\title{
THE GEOMETRY OF JULIA SETS
}

\author{
JAN M. AARTS AND LEX G. OVERSTEEGEN
}

\begin{abstract}
The long term analysis of dynamical systems inspired the study of the dynamics of families of mappings. Many of these investigations led to the study of the dynamics of mappings on Cantor sets and on intervals. Julia sets play a critical role in the understanding of the dynamics of families of mappings. In this paper we introduce another class of objects (called hairy objects) which share many properties with the Cantor set and the interval: they are topologically unique and admit only one embedding in the plane. These uniqueness properties explain the regular occurrence of hairy objects in pictures of Julia sets-hairy objects are ubiquitous. Hairy arcs will be used to give a complete topological description of the Julia sets of many members of the exponential family.
\end{abstract}

\section{INTRODUCTION}

The topological structure of the Julia sets (the unstable sets) of the quadratic family $f_{c}(z)=z^{2}+c$ and the exponential family $f_{\lambda_{1}, \lambda_{2}}(z)=\lambda_{1} e^{z}+\lambda_{2} e^{-z}$ has not yet been fully elucidated. This paper is mainly concerned with the Julia sets of exponential mappings. Our work on Julia sets in this family is based on work by Devaney and coauthors [D1, D2, D4, DKr] who had shown that the Julia set $J_{\lambda}$ of $E_{\lambda}(z)=\lambda e^{z}$ (for real $0<\lambda \leq 1 / e$ ) was a "Cantor bouquet," the closure of a particular union of sets of the form (Cantor set) $\times[0, \infty)$ in the complex plane [DKr]. Devaney observed that apparently similar Cantor bouquet Julia sets occur for a wide variety of transcendental entire functions, including $z \rightarrow \lambda \sin z$, and $z \rightarrow \lambda \cos z$ for certain complex values of the parameter $\lambda$ [DT]. It was not clear whether these Cantor bouquets were in fact homeomorphic one to another, either within a family, or across families. Compare the frequent occurrence of Cantor sets in dynamics: whether or not the dynamics are conjugate, the Cantor sets themselves are all topologically equivalent, and, in the planar case, must be equivalently embedded. We will show that these Julia sets are indeed homeomorphic and all of them are equivalently embedded in the plane. This is in spite of the fact that there are no nonzero values of $\lambda$ and $\mu$ for which $z \rightarrow \lambda e^{z}$ and $z \rightarrow \mu \sin z$ are conjugate. It also stands in contrast to a result of McMullen's which shows that the Cantor bouquets in the

Received by the editors May 22, 1991.

1980 Mathematics Subject Classification (1985 Revision). Primary 54H20, 33A10; Secondary 34C35, 54F65, 58F08.

Key words and phrases. Julia set, exponential map, model, Cantor bouquet, hairy arc.

The second author was supported in part by NSF-RII-8610669. The results of this paper were announced at the 1991 Spring Topology Conference in Sacramento, California, April 10-13, 1991. 
exponential family have Hausdorff dimension 2 and plane Lebesgue measure 0 , while those in the sine family have Hausdorff dimension 2 but positive plane Lebesgue measure [EL, McM]. We shall also present a very simple model for these Julia sets, which reveals all of its topological properties.

Our investigations of Julia sets of exponential mappings led to the notion of hairy objects. Objects with essentially the same properties have a long history preceding our interest by many decades (see for example [L]). However, the first indications that these objects are unique appears to be quite recent (cf. [BO, C]). The hairy objects introduced in this paper also share a stronger form of uniqueness: planar hairy objects of the same type are all ambiently homeomorphic. They share this strong form of uniqueness with the arc and the Cantor set and it explains their regular occurrence in pictures of Julia sets-hairy objects are ubiquitous. All planar hairy objects are closed subsets of the plane consisting of an uncountable number of half rays (referred to as hairs), which are connected either at $\infty$ or through a (base) circle or arc, such that each hair is approximated (smoothly) from two sides in the plane by other hairs. Every hairy object is locally compact and the set of endpoints of the hairs is a dense, 1-dimensional and totally disconnected set. We hope that the study of hairy objects will also shed light on the topological structure of Julia sets in the quadratic family.

The paper is organized as follows: In $\S 1$ we will study the Julia set of the exponential map $z \rightarrow \lambda e^{z}$. This leads to the notion of an hairy arc which is introduced in $\S 2$. In $\S 3$ we study hairy arcs in detail by proving their uniqueness and providing an internal characterization. In $\S 4$ we study embeddings of hairy arcs in the plane. The last section $(\S 5)$ is devoted to Julia sets of hyperbolic functions.

\section{ThE EXPONENTIAL MAP}

In this section we give a precise description of the Julia sets in the oneparameter family of exponentials $E_{\lambda}(z)=\lambda e^{z}$, where $\lambda$ is a real number with $0<\lambda \leq \frac{1}{e}$. In $\S 5$ other examples of Julia sets will be discussed. An introduction into the subject can be found in the books [D3 and DKe]. The dynamical behavior of the exponential family has been studied by various authors. The pioneering work has been done by Misiurewicz $[\mathrm{M}]$ and Devaney [D1, D2, $\mathrm{D} 4, \mathrm{DKr}]$. The result that the Julia set of $E_{\lambda}$ is the complex plane, for all $\lambda>\frac{1}{e}$, is essentially due to Misiurewicz [M]. The dynamics of the systems $E_{\lambda}, 0<\lambda \leq \frac{1}{e}$, in particular the geometry of their Julia sets $J\left(E_{\lambda}\right)$ has been revealed in [DKr and D4]. Our work in this section utilizes ideas and notions from both papers. The construction of the topological map of the Julia sets, to be presented in this paper, is a modification of the construction of the crowns in the Cantor bouquets. What is new here is the construction of special models of the Julia sets, which are called straight brushes. The construction shows that the Julia sets are "smooth compacta" (smooth in the topological sense; see 3.9 for a definition). From the results in the subsequent sections it will follow that all straight brushes are ambiently homeomorphic and, consequently, that all Julia sets are ambiently homeomorphic. This result is not only valid for the systems $E_{\lambda}, 0<\lambda \leq \frac{1}{e}$, for which the result also follows from the quasiconformal conjugacy of any two such systems [DG], but also holds for other systems which are not equivalent to a system $E_{\lambda}$ for some $\lambda$. 
1.1. We shall define straight brushes as subsets of the product $[0, \infty) \times \mathscr{P}$ of the half line and the irrational numbers. Two structures of the set $\mathscr{P}$ of irrational numbers (in $\mathscr{R}$ ) are used, namely, the product structure and the order structure. By considering partitions of $\mathscr{P}$ by means of collections $\mathscr{U}_{0}, \mathscr{U}_{1}, \mathscr{U}_{2}, \ldots$ of pairwise disjoint intervals in $\mathscr{P}$ (with rational endpoints) such that each element of $\mathscr{U}_{k}$ is the union of a doubly infinite sequence of elements of $\mathscr{U}_{k+1}, k=0,1,2, \ldots$, while mesh $\left(\mathscr{U}_{k}\right) \rightarrow 0$ as $k \rightarrow \infty$, one can see that $\mathscr{P}$ is homeomorphic to the countable infinite product $\prod_{i=0}^{\infty} \mathscr{Z}$ of copies of the integers $\mathscr{Z}$. The indexing of the partitions can be arranged in such a way that the homeomorphism between $\mathscr{P}$ and $\prod_{i=0}^{\infty} \mathscr{Z}$ is also an order isomorphism between $\mathscr{P}$ with the natural order, inherited from $\mathscr{R}$, and the product $\prod_{i=0}^{\infty} \mathscr{Z}$ with the lexicographic order. We shall use the product structure and the order structure interchangeably. In particular we identify $[0, \infty) \times \mathscr{P}$ with a subset of $\mathscr{R}^{2}$ in a natural way.

1.2. Definition. A straight brush is a subset $B$ of the set $[0, \infty) \times \mathscr{P}$ in $\mathscr{R}^{2}$ with the following properties.

a. Hairiness. For each $(y, \alpha) \in B$, there is a $t_{\alpha} \in[0, \infty)$ such that $\{t \mid(t, \alpha) \in B\}=\left[t_{\alpha}, \infty\right)$. The point $e_{\alpha}=\left(t_{\alpha}, \alpha\right)$ is the endpoint of the hair $h_{\alpha}=\left[t_{\alpha}, \infty\right) \times\{\alpha\}$ at $\alpha$.

b. Density. The set $\{\alpha \mid(y, \alpha) \in B$ for some $y\}$ is dense in $\mathscr{P}$ and, for each $(y, \alpha) \in B$, there exist sequences $\left(\beta_{n}\right)$ and $\left(\gamma_{n}\right)$ in $\mathscr{P}$ such that $\beta_{n} \uparrow \alpha, \gamma_{n} \downarrow \alpha, t_{\beta_{n}} \rightarrow t_{\alpha}$ and $t_{\gamma_{n}} \rightarrow t_{\alpha}$.

c. Compact sections. $B$ is a closed subset of $\mathscr{R}^{2}$.

1.3. Let $\lambda$ be a (fixed) real number with $0<\lambda \leq \frac{1}{e}$. Let $p_{\lambda}$ denote the unique fixed point of $E_{\lambda}$ with $p_{\lambda} \geq 1$. It is to be noted that $\left|E_{\lambda}^{\prime}(z)\right|>1$ for all $z$ with $\operatorname{Re} z>p_{\lambda}$. By standard arguments, involving Schwarz's lemma, it may be proved that the open half plane $\left\{z \mid \operatorname{Re} z<p_{\lambda}\right\}$ and the strips $\left\{z \mid\left(2 k-\frac{1}{2}\right) \pi \leq\right.$ $\left.\operatorname{Im} z \leq\left(2 k+\frac{1}{2}\right) \pi\right\}, k \in \mathscr{Z}$, are subsets of the Fatou set of $E_{\lambda}$. The rays $\left\{z \mid \operatorname{Re} z \geq p_{\lambda}, \operatorname{Im} z=2 k \pi\right\}, k \in \mathscr{Z}$, belong to the Julia set $J\left(E_{\lambda}\right)$ of $E_{\lambda}$. Recall that a ray is a closed subset of the plane, which is homeomorphic to the half line $[0, \infty)$.

We see that the rays $\left\{z \mid \operatorname{Re} z \geq p_{\lambda}, \operatorname{Im} z=k \pi\right\}, k \in \mathscr{Z}$, alternately belong to the Julia and Fatou sets. By taking preimages we find interleaved collections of rays in the Julia and the Fatou set of $E_{\lambda}$.

A large part of this section is devoted to the proving of the following main result.

1.4. Theorem. Suppose $0<\lambda \leq \frac{1}{e}$. There exists a straight brush $B$ in $[0, \infty) \times$ $\mathscr{P}$ and a homeomorphism $\varphi$ of $B$ onto the Julia set $J\left(E_{\lambda}\right)$ of $E_{\lambda}$.

The following is a preliminary definition.

1.5. Definition. For each $x \in[0, \infty)$ and $n \in \mathscr{Z}$, the square at $(x, 2 n)$ is defined by

$$
S(x, n)=\left\{\xi+i \eta \mid x \leq \xi \leq x+\pi,\left(2 n-\frac{1}{2}\right) \pi \leq \eta \leq\left(2 n+\frac{1}{2}\right) \pi\right\} .
$$

It is to be noted that $S(x, n) \cap J\left(E_{\lambda}\right) \neq \varnothing$, for all $x \geq p_{\lambda}-\pi$ and $n \in \mathscr{Z}$. 
1.6. Construction of $B$ and definition of $\varphi$. We define a straight brush $B$ in $[0, \infty) \times \mathscr{P}$ and a continuous map $\varphi$ of $B$ into $J\left(E_{\lambda}\right)$. The additional properties of $\varphi$ will become apparent later on. Let $x \geq p_{\lambda}$ and $\alpha \in \mathscr{P}$. Using the product representation of $\mathscr{P}$ we let $\alpha=\left(n_{0}, n_{1}, n_{2}, \ldots\right)$ with $n_{i} \in \mathscr{Z}$. We shall decide whether or not $(x, \alpha) \in B$. If $(x, \alpha) \in B$, the image $\varphi(x, \alpha)$ is defined in such a way that

$$
\left(2 n_{k}-\frac{1}{2}\right) \pi<\operatorname{Im}\left(E_{\lambda}^{k}(\varphi(x, \alpha))\right)<\left(2 n_{k}+\frac{1}{2}\right) \pi .
$$

Inductively on $k$ we define real numbers $x_{k}$ and squares $R\left(x_{k}, n_{k}\right)$. We let $x_{0}=x$ and $R\left(x_{0}, n_{0}\right)=S\left(x, n_{0}\right)$. Suppose that $x_{l}$ and $R\left(x_{l}, n_{l}\right)$ have been defined for $l \leq k$. We consider two cases.

(1) $R\left(x_{k}, n_{k}\right) \neq \varnothing$ and there exists $\xi$ with

$$
S\left(\xi, n_{k+1}\right) \subset E_{\lambda}\left(R\left(x_{k}, n_{k}\right)\right) \text {. }
$$

Let $\xi_{m}=\min \{\xi \mid \xi$ satisfies $(*)\}$. If $\xi_{m} \geq p_{\lambda}$, we put $x_{k+1}=\xi_{m}$ and

$$
R\left(x_{k+1}, n_{k+1}\right)=S\left(\xi_{m}, n_{k+1}\right) .
$$

If $\xi_{m}<p_{\lambda}$, we let $x_{k+1}=x_{k}$ and $R\left(x_{k+1}, n_{k+1}\right)=\varnothing$.

(2) If case (1) does not apply, we let $x_{k+1}=x_{k}$ and $R\left(x_{k+1}, n_{k+1}\right)=\varnothing$.

For each $k \in \mathscr{N}$, let $B_{k}=E_{\lambda}^{-k}\left(R\left(x_{k}, n_{k}\right)\right)$, where we choose the branch of $E_{\lambda}^{-k}$ such that $B_{k} \subset B_{0}$. The straight brush $B$ is defined by

$$
B=\left\{(x, \alpha) \mid R\left(x_{k}, n_{k}\right) \neq \varnothing, \text { for all } k\right\} .
$$

For each $(x, \alpha) \in B$, we define

$$
\varphi(x, \alpha)=\bigcap\left\{B_{k} \mid k=0,1,2, \ldots\right\} .
$$

It is to be noted that $B_{k+1} \subset B_{k} \subset \cdots \subset B_{0}$, for $k>0$, and that $\operatorname{diam}\left(B_{k}\right) \rightarrow 0$ as $k \rightarrow \infty$, since $\left|E_{\lambda}^{\prime}\right|>1$ in the half plane $\operatorname{Re} z>p_{\lambda}$. It follows that $\varphi$ is a well-defined map of $B$ into $\mathscr{C}$.

1.7. Lemma. $B$ is a straight brush and $\varphi$ is an injective continuous map of $B$ into the Julia set $J\left(E_{\lambda}\right)$.

Proof. First observe that, for any square $S(x, n)$ with $x \geq p_{\lambda}, E_{\lambda}(S(x, n))$ is an annular region, centered at the origin, with width $\geq 22$, while the diagonal of any square $S(y, m)$ has length $\leq 4.5$. The proof consists of six parts.

1. First we show that $\varphi$ is one-to-one. Suppose that $(x, \alpha)$ and $(y, \beta)$ are distinct points of $B$. Let $\alpha=\left(n_{0}, n_{1}, \ldots\right)$ and $\beta=\left(m_{0}, m_{1}, \ldots\right)$. If $\alpha \neq \beta$, then $n_{k} \neq m_{k}$ for some $k$, whence $R\left(x_{k}, n_{k}\right) \cap R\left(y_{k}, m_{k}\right)=\varnothing$. If $\alpha=\beta$, then $x \neq y$, and we may assume $x<y$. Then $x_{k}<y_{k}$, for all $k$, as is easily seen, and in fact $y_{k}-x_{k} \rightarrow \infty$ as $k \rightarrow \infty$. Hence there exists $k$ such that $R\left(x_{k}, n_{k}\right) \cap R\left(y_{k}, m_{k}\right)=\varnothing$.

2. The continuity of $\varphi$ follows from the following observation. Let $(x, \alpha)$ $\in B$ and $\alpha=\left(n_{0}, n_{1}, n_{2}, \ldots\right)$. If $\beta=\left(m_{0}, m_{1}, \ldots\right) \in \mathscr{P}$ has $n_{i}=m_{i}$, for all $i \leq k$, then we can choose $\delta>0$ such that $|y-x|<\delta$ and $(y, \beta) \in B$ imply $R\left(x_{i}, n_{i}\right) \cap R\left(y_{i}, m_{i}\right) \neq \varnothing$, for all $i \leq k$. It follows that $\varphi(x, \alpha)$ is close to $\varphi(y, \beta)$.

3. Let $(x, \alpha) \in B$ and $\alpha=\left(n_{0}, n_{1}, n_{2}, \ldots\right)$. We have $R\left(x_{k}, n_{k}\right) \cap$ $J\left(E_{\lambda}\right) \neq \varnothing$ for all $k$. It follows that $d\left(\varphi(x, \alpha), J\left(E_{\lambda}\right)\right)=0$, whence $\varphi(x, \alpha) \in$ $J\left(E_{\lambda}\right)$. 
4. We discuss the hairiness of $B$. Let $(x, \alpha) \in B$ with $\alpha=\left(n_{0}, n_{1}, \ldots\right)$ and suppose $x<y$. We show that $(y, \alpha) \in B$. By induction it follows that $x_{k}<y_{k}$ and $R\left(y_{k}, n_{k}\right) \neq \varnothing$, for all $k$. Consequently, $(y, \alpha) \in B$. Now suppose that $(z, \alpha) \notin B$. Consider the smallest $k$ with $R\left(z_{k}, n_{k}\right) \neq$ $\varnothing$, but $R\left(z_{k+1}, n_{k+1}\right)=\varnothing$ Then, either there exists no $\xi$ with $S\left(\xi, n_{k+1}\right) \subset$ $E_{\lambda}\left(R\left(z_{k}, n_{k}\right)\right)$ or there does exist such a $\xi$, but for the minimal $\xi_{m}$ with that property, we have $\xi_{m}<p_{\lambda}$. In both cases the same holds true for all $y$ slightly larger than $z$. It follows that $\{x \mid(x, \alpha) \in B\}$ is of the form $\left[t_{\alpha}, \infty\right)$.

5. As for the density of $B$, that $\{\alpha \mid(y, \alpha) \in B$ for some $y\}$ is dense in $\mathscr{P}$ follows from the observation in 1.3 about the interleaved collections of rays in the Julia and the Fatou set. Now suppose $(x, \alpha) \in B$. Let $\alpha=$ $\left.\left(n_{0}, n_{1}, n_{2}, \ldots\right)\right)$. Choose a $y$ which is slightly bigger than $x$. Then the inner radius of $E_{\lambda}\left(R\left(y_{k-1}, n_{k-1}\right)\right)$ will be much bigger than the inner radius of $E_{\lambda}\left(R_{k-1}\left(x_{k-1}, n_{k-1}\right)\right.$, for $k$ sufficiently large. It follows that $(y, \beta) \in B$ for $\beta=\left(n_{0}, n_{1}, \ldots, n_{k-1}, n_{k} \pm 1,0,0, \ldots\right)$.

6. It remains to prove that $B$ is a closed subset of $R^{2}$. Suppose that $(x, s) \notin B$. We shall construct an open rectangle $S$ such that $(x, s) \in S \subset$ $R^{2} \backslash B$. We only discuss the case that $s$ is rational, as that is the more difficult case (for the case that $s$ is irrational, see also 4 above).

Consider the minimal $k$ such that $s \notin \cup \mathscr{U}_{k}$ (notation of 1.1). There is a unique $n_{k}$ such that, for all $m \in \mathscr{Z},\left(n_{0}, n_{1}, \ldots, n_{k}, m, \ldots\right)<s<$ $\left(n_{0}, n_{1}, \ldots, n_{k-1}, n_{k}+1, m, \ldots\right)$. A close look at the definition of $B$ reveals that we can choose $N$ so large that no point $(x, \gamma)$ belongs to $B$, where $\gamma$ has the form

$$
\begin{aligned}
& \gamma=\left(n_{0}, \ldots, n_{k-1}, n_{k}, m, \ldots\right), \text { for some } m \geq N, \text { or } \\
& \gamma=\left(n_{0}, \ldots, n_{k-1}, n_{k}+1, m, \ldots\right), \text { for some } m \leq-N .
\end{aligned}
$$

For any such $\gamma$, we have $R\left(x_{k+1}, m\right)=\varnothing$. It also follows that there is a $\delta>0$ such that, for all $y$ with $|x-y|<\delta$, we have $R\left(y_{k+1}, m\right)=\varnothing$, for all $m>N$ and all $m<-N$. Let $\sigma=\left(n_{0}, \ldots, n_{k}, N, 0, \ldots\right)$, and $\tau=$ $\left(n_{0}, \ldots, n_{k-1}, n_{k}+1,-N, 0, \ldots\right)$ and $S=\{(y, t)|| x-y \mid<\delta, \sigma<t<\tau\}$. Then $(x, s) \in S \subset R^{2} \backslash B$.

We define the inverse of $\varphi$, which is named $\psi$.

1.8. Construction of $\psi: J\left(E_{\lambda}\right) \rightarrow B$. Let $z \in J\left(E_{\lambda}\right)$. For each $k \in \mathcal{N}$, there is a unique $n_{k} \in \mathscr{Z}$ such that

$$
\left(2 n_{k}-\frac{1}{2}\right) \pi<\operatorname{Im} E_{\lambda}^{k}(z)<\left(2 n_{k}+\frac{1}{2}\right) \pi .
$$

This defines the itinerary $n(z)=\left(n_{0}, n_{1}, n_{2}, \ldots\right)$ of $z$. For each $k \in \mathcal{N}$, we let $R_{k}^{k}=S\left(u, n_{k}\right)$, where $u$ is minimal with respect to the properties $u \geq p_{\lambda}$ and $E_{\lambda}^{k}(z) \in R_{k}^{k}$. For each $k \in N$, we define $R_{i}^{k}$ for $i=k$ to $i=0$ inductively as follows. If $R_{l}^{k}$ has been defined, we let $R_{l-1}^{k}=S\left(v, n_{l-1}\right)$, where $v$ is maximal with respect to the property $R_{l}^{k} \subset E_{\lambda}\left(R_{l-1}^{k}\right)$. Note that the last condition ensures that the square $R_{l}^{k}$ hits the inner radius of the semiannulus $E_{\lambda}\left(R_{l-1}^{k}\right)$ in exactly one point. For each $k \in \mathcal{N}$, we define $x_{k}$ by the condition $R_{0}^{k}=S\left(x_{k}, n_{0}\right)$.

It is to be noted that $z \in R_{0}^{k}$, for each $k \in \mathscr{N}$. It follows that

$\operatorname{Re} z-\pi \leq x_{0} \leq \operatorname{Re} z$, and $x_{0} \leq x_{k} \leq x_{k+1} \leq \operatorname{Re} z+\pi, \quad$ for all $k$. 
Finally we define

$$
x^{\infty}=\lim _{k \rightarrow \infty} x_{k} \text { and } \psi(z)=\left(x^{\infty}, n(z)\right) .
$$

1.9. Lemma. $\varphi \circ \psi=\operatorname{id}_{J\left(E_{\lambda}\right)}$.

Proof. We use the notation of 1.8. Define $R_{0}^{\infty}=S\left(x^{\infty}, n_{0}\right)$. We have $R_{0}^{\infty}=$ $\lim _{k \rightarrow \infty} R_{0}^{k}$ in the Hausdorff metric. For $l=1,2, \ldots, R_{l}^{\infty}=\lim _{k \rightarrow \infty} R_{l}^{k}$. As follows from the observation in 1.8, by the continuity of $E_{\lambda}$ the square $R_{l}^{\infty}$ hits the inner radius of the annulus $E_{\lambda}\left(R_{l-1}^{\infty}\right)$ in exactly one point $w_{l}$. It is to be noted that $\operatorname{Re} w_{l} \geq p_{\lambda}$. Using these observations we compute $\varphi(\psi(z))$ for a $z \in J\left(E_{\lambda}\right)$. Applying the construction of 1.6 we find

$$
R\left(x_{k}^{\infty}, n_{k}(z)\right)=R_{k}^{\infty}, \quad k \in \mathscr{N},
$$

and

$$
\varphi(\psi(z))=\varphi\left(x^{\infty}, n(z)\right) .
$$

It follows that $\psi(z) \in B$ and $E_{\lambda}^{k}\left(\varphi(\psi(z)) \in R_{k}^{\infty}, k \in \mathscr{N}\right.$. Consequently

$$
\varphi(\psi(z))=\varphi\left(x^{\infty}, n(z)\right)=\bigcap\left\{E_{\lambda}^{-k}\left(R_{k}^{\infty}\right) \mid k \in \mathscr{N}\right\}=z .
$$

1.10. Lemma. The map $\varphi$ is a homeomorphism from $B$ onto $J\left(E_{\lambda}\right)$ with inverse $\psi$.

Proof. This follows in a standard fashion from Lemmas 1.7 and 1.9 once we have noticed that $\psi$ sends bounded sets to bounded sets.

Theorem 1.4 has an interesting corollary. Using the product representation of $\mathscr{P}$ the shift $\sigma: \mathscr{P} \rightarrow \mathscr{P}$ is defined by $\sigma\left(n_{0}, n_{1}, \ldots\right)=\left(n_{1}, n_{2}, \ldots\right)$. The shift induces a map $\sigma^{*}$ on the endpoints of the straight brush $B$ as follows:

$$
\sigma^{*}\left(l_{\alpha}\right)=\sigma^{*}\left(t_{\alpha}, \alpha\right)=\left(t_{\sigma(\alpha)}, \sigma(\alpha)\right) .
$$

1.11. Corollary. The map $\varphi$ is a conjugation of $\sigma^{*}$ to the restriction of $E_{\lambda}$ to the set of endpoints of $J\left(E_{\lambda}\right)$.

In condition $\mathrm{b}$ of Definition 1.2 the order of the irrationals $\mathscr{P}$ is involved. To capture the order in a topological way we shall attach the hairs of the brush to an arc, which will be called the base of the brush. We shall do this by means of a suitable compactification of $\mathscr{C}$.

1.12. Compactifying the brush. The most natural way of compactifying $\mathscr{E}$ is undoubtedly the one-point compactification. After identification of $\mathscr{R}^{2}$ with $\mathscr{C}$ the one-point compactification of $\mathscr{C}$ entails that of the straight brush $B$. The resulting space is a smooth Cantor bouquet [BO]. A serious drawback of this compactification is that not all embeddings of the Cantor bouquet in the plane are topologically equivalent. Specifically, a variation of condition 1.2.b may fail to hold in countably many points. For this reason we shall describe another construction by which $\mathscr{C}$ is compactified to a topological disc.

We let $\mathscr{R}^{*}$ denote the extended real numbers- $\mathscr{R}^{*}=\{-\infty\} \cup \mathscr{R} \cup\{\infty\}-$ with the usual order and topology. Identifying $\mathscr{R}^{2}$ and $\mathscr{C}$, we can compactify $\mathscr{C}$ by $\mathscr{R}^{*} \times \mathscr{R}^{*}$. The set $E=\operatorname{cl}_{\mathscr{R}^{*} \times \mathscr{R}} \cdot B$ is called a straight hairy arc. In this compactification $E$ of $B$ the hairs of $B$ are attached to the base $\{\infty\} \times \mathscr{R}^{*}$ of $E$ (or $B$ ). Topological copies of $E$ are called hairy arcs. These are the objects to be studied in the subsequent sections of the paper. 
We shall indicate how to compactify $\mathscr{C}$ such that $J\left(E_{\lambda}\right)\left(0<\lambda \leq \frac{1}{e}\right)$ compactifies to a hairy arc. To this end we describe an embedding of $\mathscr{C}\left(\mathscr{R}^{2}\right)$ into $\mathscr{R}^{*} \times \mathscr{R}^{*}$. The embedding of $\mathscr{R} \times \mathscr{R}$ into $\mathscr{R}^{*} \times \mathscr{R}^{*}$ is the natural one with the following exception. The edge $\left\{(\infty, t) \mid t \in \mathscr{R}^{*}\right\}$ is attached to $\mathscr{C}$ in a different way, which is conveniently described by specifying a collection of open sets containing neighborhood bases for all points of this edge. By Theorem 1.4 there exist a straight brush $B \subset[0, \infty) \times \mathscr{P}$ and a homeomorphism $\varphi: B \rightarrow J\left(E_{\lambda}\right)$. Denote by $D$ the dense subset $\{\alpha \mid(y, \alpha) \in B$ for some $y\}$ of $\mathscr{P}$. For each $\alpha \in D$ we define the open sets of the following forms:

1. $\left\{(\infty, t) \mid t>\alpha, t \in \mathscr{R}^{*}\right\}$ together with the points in the points in $\mathscr{R} \times \mathscr{R}^{*}$ which lie above the ray $\varphi\left(\left[t_{\alpha}, \infty\right) \times\{\alpha\}\right)$ and to the right of $\{u+i y \mid y \in$ $\left.\mathscr{R}^{*}\right\}$, where $u>t_{\alpha}+\pi$.

2. Similarly as in 1, but taking the union of $\left\{(\infty, t) \mid t<\alpha, t \in \mathscr{R}^{*}\right\}$ and the set of points in $\mathscr{R} \times \mathscr{R}^{*}$ which lie under the ray $\varphi\left(\left[t_{\alpha}, \infty\right) \times\{\alpha\}\right)$ and to the right of the half-line.

The collection of all finite intersections of sets obtained in this way contains the neighborhood bases for the points of the edge $\left\{(\infty, t) \mid t \in \mathscr{R}^{*}\right\}$. In this way we have defined an embedding of $\mathscr{R}^{2}$ in $\mathscr{R}^{*} \times \mathscr{R}^{*}$. The closure of $J\left(E_{\lambda}\right)$ in this compactification is easily seen to be homeomorphic to the straight hairy arc $E$, related to $B$ (see $\S 2$ ).

\section{HAIRY ARCS}

In this section we shall define the notion of a hairy arc (abbreviated ha). This will be accomplished by first giving a geometric definition of a straight one-sided hairy arc (abbreviated sosha) in the plane. A ha will be defined to be any topological image of a sosha. It will follow from the results in $\S 3$ that all soshas (and hence all hairy arcs) are homeomorphic. Moreover we shall show in $\S 4$ that, under mild conditions, all hairy arcs are equivalently embedded in the plane. Our exposition follows the pattern that sometimes is used for the discussion of the arc or the Cantor set, namely, that of geometric definition, prototype, uniqueness and tameness.

The unit interval is denoted by $I$.

2.1. Definitions. A straight one-sided hairy arc (abbreviated sosha) $X$ is a compact subset of the unit square $I^{2}$ of the following form. There exists a function $l: I \rightarrow I$, called the length function, such that

1. For all $(x, y) \in I^{2}$ we have $(x, y) \in X$ if and only if $0 \leq y \leq l(x)$;

2. The sets $\{x \in I \mid l(x)>0\}$ and $\{x \in I \mid l(x)=0\}$ are both dense in $[0,1]$ and $l(0)=l(1)=0$.

3. For each $x \in I$ with $l(x)>0$ there exist sequences $\left(p_{n}\right)$ and $\left(q_{n}\right)$ in $I$ such that $p_{n} \uparrow x, q_{n} \downarrow x$ and $\lim l\left(p_{n}\right)=\lim l\left(q_{n}\right)=l(x)$.

For each $x \in I$, the set $\{(x, y) \mid 0 \leq y \leq l(x)\}$ is called the hair at $x$. The set $\{(x, y) \mid(x, y) \in X, y>0\}$ is called the straight brush of the sosha. The set $I \times\{0\}$ is called the base.

We have already encountered a sosha in 1.12 . Identifying $\mathscr{R}^{*}$ with $I$, it can be seen that $E=\operatorname{cl}_{\mathscr{R}} \times \mathscr{R} * B$ is a sosha and that $B$ in the straight brush of the sosha. A simple construction of a sosha follows. 
2.2. Prototype of a sosha. The sosha $X$ is constructed as the intersection of a sequence $\left(T_{n}\right)$ of subsets of $I^{2}$, all containing the base $I \times\{0\}$. Each $T_{n}$ will be a finite union of rectangles $R(n, j)$ with sides parallel to the $x$ - and $y$-axis and intersecting each other at most in a subset of a vertical edge. In each rectangle $R(n, j)$ we will select $(2 n+1)$ rectangles $R(n, j, k) . T_{n+1}$ will be the union of all these rectangles $R(n, j, k)$, which will be relabeled as $R(n+1, j)$. The desired prototype is $X=\bigcap\left\{T_{n} \mid n=0,1,2, \ldots\right\}$ (see Figure 1).

Let $T_{0}=T_{1}=I^{2}$. Partition the base $I \times\{0\}$ of $T_{1}$ into three intervals of equal length, labeled $I_{1}, I_{2}, I_{3}$ from left to right. Over each interval $I_{j}, j=$ $1,2,3$, erect a rectangle of height $\frac{1}{2}, 1$ and $\frac{1}{2}$ from left to right, respectively. Suppose $T_{n}$ is a finite union of rectangles $R(n, j)$ whose bases $B(n, j)$ partition $[0,1]$ into intervals of equal length. Let $h(n, j)$ denote the height of rectangle $R(n, j)$. Partition each interval $B(n, j)$ into $(2 n+1)$ intervals of equal length labeled $I(n, j, k), k=1, \ldots, 2 n+1$, from left to right. Erect over each interval $I(n, j, k)$ a rectangle $R(n, j, k)$ of height $\frac{n+1-|n+1-k|}{n+1} \cdot h(n, j)$. Put $T_{n+1}=\bigcup R(n, j, k)$ and $X=\bigcap\left\{T_{n} \mid n=0,1,2, \ldots\right\}$. Note that the center line of each rectangle will be a hair contained in $X$. It is easily verified that $X$ is a sosha. In particular, since each $T_{n}$ is a continuum and $T_{n+1} \subset T_{n}, X$ is a continuum. We will refer to the set $D=\{(x, 0) \mid 0 \leq x \leq 1\}$ as the base of the sosha $X$.

2.3. Definition. A hairy arc (abbreviated ha) is any topological image of any sosha. A one-sided hairy arc (abbreviated osha) is an embedding $\varphi$ of a hairy

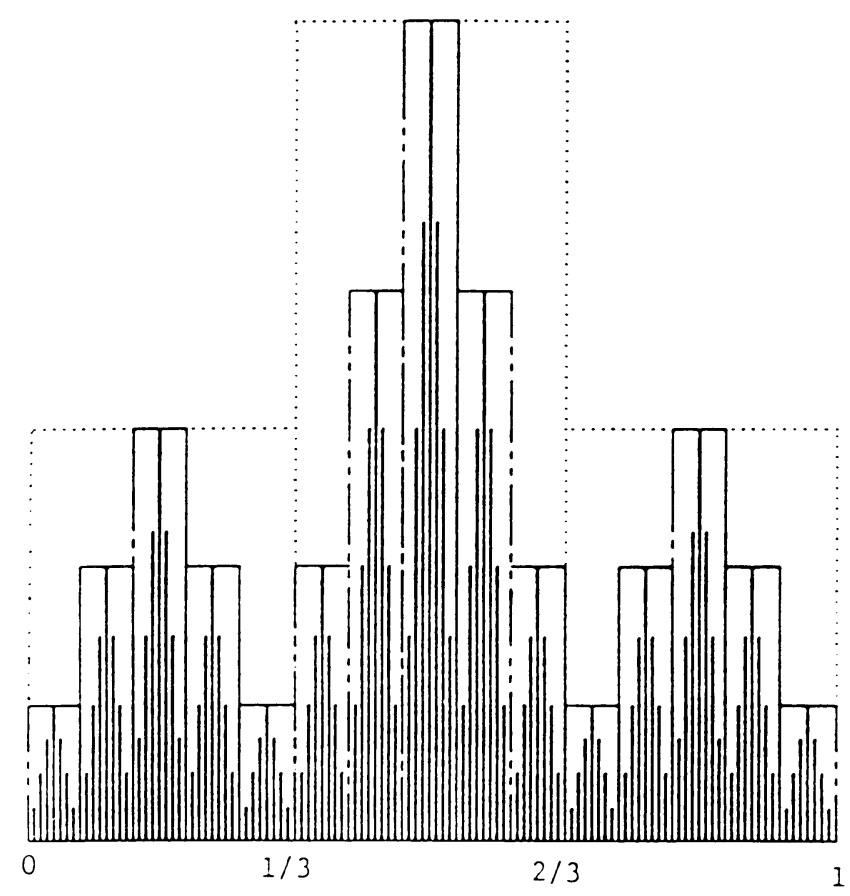

FIGURE 1 
arc $X$, with base $D$, into the plane such that all hairs are attached to the same side of the base $\varphi(D)$.

It is to be noted that there are no hairs attached to the endpoints of the base $D$. Therefore it is not difficult to define the "sides" of $\varphi(D)$ in the plane. We collect some basic properties of soshas.

2.4. Proposition. Let $l$ be the length function of a sosha $X$. The function $l$ is upper semicontinuous. Let $[a, b]$ be any subinterval of $[0,1]$. The function $l$ attains a maximum $M$ on $[a, b]$ and assumes on $[a, b]$ all values between 0 and $M$.

Proof. The upper semicontinuity of $l$ is an easy consequence of the compactness of $X$. In a standard fashion it follows that $l$ is bounded. Let $M=\sup \{l(x) \mid x \in[a, b]\}$. From the compactness of $E$ it follows that the value $M$ is attained by $l$. For each $c \in[0, M)$ the set $C=\{x \mid l(x)>c\}$ is nonempty and, by 3 of Definition 2.1, dense in itself. It follows that $\operatorname{cl}(C)$ is a Cantor set. As any Cantor set in $[0,1]$ has endpoints, by way of contradiction it follows from 3 of Definition 2.1 that the set of values attained by $l$ on these endpoints equals $\{c\}$.

We omit the proof of the following corollary.

2.5. Corollary. The set (of endpoints) $Q=\{(x, l(x)) \mid l(x)>0\}$ is dense in the sosha $X$. The set $Q \cup(I \times\{0\})$ is connected.

\section{UNIQUENESS AND CHARACTERIZATION OF THE HAIRY ARC}

In this section we shall show that any two soshas, considered as subsets of $\mathscr{R}^{2}$, are ambiently homeomorphic. It follows that any two hairy arcs are homeomorphic.

3.1. We shall consider special partitions of the first factor of the unit square. Recall that a partition $P$ of $[0,1]$ is a finite subset $\left\{x_{0}, \cdots, x_{k}\right\}$ of $[0,1]$ such that $0=x_{0}<x_{1}<\cdots<x_{k}=1$. If $P_{1}$ and $P_{2}$ are partitions and $P_{1} \subset P_{2}$, then $P_{2}$ in called a refinement of $P_{1}$. Let $E$ be a sosha (Definition 2.1). The partitions $P=\left\{x_{0}, \ldots, x_{k}\right\}$ to be considered in the sequel have the additional property that $l\left(x_{i}\right)=0,0 \leq i \leq k$. We define $M_{i}=\max \left\{l(x) \mid x_{i-1} \leq x \leq\right.$ $\left.x_{i}\right\}, i=1, \ldots, k$. For each $i \in\{1, \ldots, k\}$ we pick a $c_{i} \in\left[x_{i-1}, x_{i}\right]$ such that $l\left(c_{i}\right)=M_{i}$. The bump function $b$ related to the partition $P$ and the length function $l$ is defined as follows. Let $x \in\left[x_{i-1}, x_{i}\right]$. Then $x \in\left[x_{i-1}, c_{i}\right]$ or $x \in$ $\left[c_{i}, x_{i}\right]$. In the first case we define

$$
b(x)=\max \left\{l(\xi) \mid \xi \in\left[x_{i-1}, x\right]\right\} .
$$

If $x \in\left[c_{i}, x_{i}\right]$, we let

$$
b(x)=\max \left\{l(\xi) \mid \xi \in\left[x, x_{i}\right]\right\} .
$$

It is to be observed that the bump function is independent of the choices of the $c_{i}, i=1, \ldots, k$. Note that the bump function is piecewise monotone. By Proposition 2.4 the bump function is continuous. When sequences of partitions are to be considered, we shall use two lower indices, the first index referring to the ordinal number of the partition. 
The following theorem says that there is only one sosha up to homeomorphism. So the straight hairy arc of 1.12 and the prototype of 2.2 are homeomorphic.

3.2. Theorem. Let $E_{1}$ and $E_{2}$ be soshas. Then there is a homeomorphism $\phi: \mathscr{R}^{2} \rightarrow \mathscr{R}^{2}$ such that $\phi\left(E_{1}\right)=E_{2}$. In other words, $E_{1}$ and $E_{2}$ are ambiently homeomorphic.

Proof. Let $E_{1}$ and $E_{2}$ be soshas. We shall use upper indices 1 and 2 to distinguish between mathematical objects in $E_{1}$ and $E_{2}$. Without loss of generality we may assume that

$$
\max \left\{l^{s}(x) \mid x \in[0,1]\right\}=\frac{1}{2}, \quad s=1,2 .
$$

The proof is broken down in several lemmas.

3.3. Lemma. For $s=1,2$ there are sequences $\left(P_{n}^{s}\right)$ of partitions $P_{n}^{s}=\left\{x_{n, 0}^{s}\right.$, $\left.\ldots, x_{n, k_{n}}^{s}\right\}$ such that the following properties hold.

$$
\begin{gathered}
P_{n+1}^{s} \text { is a refinement of } P_{n}^{s}, \quad n \in \mathscr{N}, s=1,2 . \\
\operatorname{mesh}\left(P_{n}^{s}\right)<\frac{1}{(n+1)^{2}}, \quad n \geq 2, s=1,2 .
\end{gathered}
$$

(3) The sequences $\left(P_{n}^{1}\right)$ and $\left(P_{n}^{2}\right)$ are isomorphic in the following sense: for each $n \in \mathscr{N}$ the partitions $P_{n}^{1}$ and $P_{n}^{2}$ have the same number of elements, namely, $k_{n}$, and for each $i \in\left\{1, \ldots, k_{n+1}\right\}$ and $j \in\left\{1, \ldots, k_{n}\right\}$,

$$
\left[x_{n+1, i-1}^{1}, x_{n+1, i}^{1}\right] \subset\left[x_{n, j-1}^{1}, x_{n, j}^{1}\right] \text { iff }\left[x_{n+1, i-1}^{2}, x_{n+1, i}^{2}\right] \subset\left[x_{n, j-1}^{2}, x_{n, j}^{2}\right] \text {. }
$$

(4) For each $n \in \mathscr{N}$, if $\left[x_{n+1, i-1}^{s}, x_{n+1, i}^{s}\right] \subset\left[x_{n, j-1}^{s}, x_{n, j}^{s}\right]$ for some $i$ and $j$, then

$$
1-\frac{1}{(n+2)^{2}}<\frac{M_{n+1, i}^{2}}{M_{n, j}^{2}}: \frac{M_{n+1, i}^{1}}{M_{n, j}^{1}}<1+\frac{1}{(n+2)^{2}} .
$$

Remark. From 4, in an elementary way, the following formulas follow.

(5) For $n, i$ and $j$ as in 4,

$$
\left(1-\frac{1}{(n+2)^{2}}\right) \frac{M_{n, j}^{2}}{M_{n, j}^{1}}<\frac{M_{n+1, i}^{2}}{M_{n+1, i}^{1}}<\left(1+\frac{1}{(n+2)^{2}}\right) \frac{M_{n, j}^{2}}{M_{n, j}^{1}} .
$$

(6) For all $n$ and $i$ with $1 \leq i \leq k_{n}$,

$$
0.5<\frac{M_{n, i}^{2}}{M_{n, i}^{1}}<1.91 \text {. }
$$

Proof. The proof is by induction on $n$. For $s=1,2$, we let $P_{0}^{s}=\{0,1\}$. We have made the assumption $M_{0,1}^{1}=M_{0,1}^{2}=\frac{1}{2}$. We assume that $P_{0}^{s}, \ldots, P_{n}^{s}$, $s=1,2$, have been constructed. We assume that $n$ is even. For odd $n$ the construction is similar with interchange of 1 and 2. The partition $P_{n+1}^{2}$ is a refinement of $P_{n}^{2}$ such that

$$
\operatorname{mesh}\left(P_{n+1}^{2}\right)<\frac{1}{(n+3)^{2}} .
$$


The reason for using $\frac{1}{(n+3)^{2}}$ instead of $\frac{1}{(n+2)^{2}}$ is that in the next step we have no control over the mesh of $P_{n+2}^{2}$. The number of elements in $P_{n}^{2}$ and $P_{n+1}^{2}$ is denoted by $k_{n}$ and $k_{n+1}$ respectively. We shall define the partition $P_{n+1}^{1}$. Let $1 \leq t \leq k_{n}$. As $P_{n+1}^{2}$ is a refinement of $P_{n}^{2}$ there are $u$ and $v$ with $1 \leq u, v \leq k_{n+1}$ such that

$$
x_{n+1, u}^{2}=x_{n, t-1}^{2} \text { and } x_{n+1, v}^{2}=x_{n, t}^{2} .
$$

The points $x_{n, t-1}^{1}$ and $x_{n, t}^{1}$ belong to $P_{n}^{1}$. We define

$$
x_{n+1, u}^{1}=x_{n, t-1}^{1} \text { and } x_{n+1, v}^{1}=x_{n, t}^{1} .
$$

We shall define the points $x_{n+1, r}^{1}$ for $u<r<v$. For $s=1,2$, pick points $c_{n, t}^{s}$ from $\left[x_{n, t-1}^{s}, x_{n, t}^{s}\right]$ with $l^{s}\left(c_{n, t}^{s}\right)=M_{n, t}^{s}$. There is a unique $w>u$ such that

$$
x_{n+1, u}^{2}<\cdots<x_{n+1, w-1}^{2}<c_{n, t}^{2}<x_{n+1, w}^{2}<\cdots<x_{n+1, v}^{2} .
$$

(If $w=u+1$ or $w=v-1$, the sequence is a little bit different.) By induction on $r$ from $u+1$ up to $w-1$ and from $v-1$ backwards to $w$ we shall define the $x_{n+1, r}^{1}$. For notational convenience we discuss only the construction of $x_{n+1, u+1}^{1}$. For $r=u+1$ we argue as follows. Consider the bump function on $\left[x_{n+1, u}^{1}, c_{n, t}^{1}\right]$. There exists $y_{n+1, u+1}^{1}$ such that

(i) $x_{n+1, u}^{1}<y_{n+1, u+1}^{1}<c_{n, t}^{1}$, and

(ii) $1-\frac{1}{(n+2)^{2}}<\frac{l^{1}\left(y_{n+1, u+1}^{1}\right)}{M_{n, t}^{1}}: \frac{M_{n+1, u+1}^{2}}{M_{n, l}^{2}}<1+\frac{1}{(n+2)^{2}}$.

By slightly moving $y_{n+1, u+1}^{1}$ to the right we find $x_{n+1, u+1}^{1}$ such that

(iij) $x_{n+1, u}^{1}<x_{n+1, u+1}^{1}<c_{n, t}^{1}$ and $l^{1}\left(x_{n+1, u+1}^{1}\right)=0$.

(iv) The inequality (4) is satisfied for $n, i=u+1$ and $j=t$.

We are going to construct a homeomorphism $\phi$ of the unit square $[0,1] \times$ $[0,1]$ onto itself such that $\phi\left(E_{1}\right)=E_{2}$. From the construction of $\phi$ it will be obvious that $\phi$ can be extended to a homeomorphism of $\mathscr{R}^{2}$ onto itself. The homeomorphism $\phi$ is obtained as composition of $\phi_{1}$ and $\phi_{2}$. The homeomorphism $\phi_{1}$ affects only the first coordinate and the homeomorphism $\phi_{2}$ affects only the second coordinate.

The following lemma is a preparation for the definition of $\phi_{1}$.

3.4. Lemma. There is a unique continuous map $\varphi:[0,1] \rightarrow[0,1]$ such that $\varphi\left(x_{n, i}^{1}\right)=x_{n, i}^{2}$ for all $n \in \mathcal{N}$ and all $i \in\left\{0, \ldots, k_{n}\right\}$. The map $\varphi$ is a uniform equivalence. For each $x \in[0,1], l^{1}(x)=0$ if and only if $l^{2}(\varphi(x))=0$.

Proof. By defining $\varphi\left(x_{n, i}^{1}\right)=x_{n, i}^{2}$, for $n \in N$ and $i \in\left\{0, \ldots, k_{n}\right\}$, we get an order preserving map of $\left\{x_{n, i}^{1} \mid n \in \mathcal{N}, i \in\left\{0, \ldots, k_{n}\right\}\right\}$ onto $\left\{x_{n, i}^{2} \mid n \in\right.$ $\left.\mathcal{N}, i \in\left\{0, \ldots, k_{n}\right\}\right\}$. That $\varphi$ is order preserving follows from (3) in Lemma 3.3. Because these sets are dense, there is a unique continuous extension to a map $\varphi:[0,1] \rightarrow[0,1]$. That $\varphi$ is a uniform equivalence follows from condition 2 in Lemma 3.3.

For any $x$ which is not a point of any partition, there is a unique sequence $\left(x_{n, i_{n}}^{1}\right)$ such that $x \in\left[x_{n, i_{n}-1}^{1}, x_{n, i_{n}}^{1}\right], n \in \mathscr{N}$. It is to be observed that $l^{1}(x)=$ $\lim _{n \rightarrow \infty} M_{n, i_{n}}^{1}$, as $l^{1}$ is upper-semicontinuous and $\operatorname{mesh}\left(P_{n}^{1}\right) \rightarrow 0$ as $n \rightarrow \infty$. By the construction of $\varphi$ as an extension to the order completion we have 
$\varphi(x) \in\left[x_{n, i_{n}-1}^{2}, x_{n, i_{n}}^{2}\right], n \in \mathcal{N}$, and $l^{2}(\varphi(x))=\lim _{n \rightarrow \infty} M_{n, i_{n}}^{2}$. From (6) following Lemma 3.3 we get

$$
l^{1}(x)=0 \text { if and only if } l^{2}(\varphi(x))=0 .
$$

For the construction of $\phi_{2}$ we shall use a type of map which is described in the following lemma.

3.5. The bump shaping lemma. Let $l$ be the length function of some sosha. We assume $l(x) \leq \frac{1}{2}$ for all $x \in[0,1]$. Let $P=\left\{x_{0}, \ldots, x_{n}\right\}$ be a partition of $[0,1]$ such that $l\left(x_{i}\right)=0, i=0, \ldots, n$ and let $b$ be its bump function. Let $\gamma_{1}, \ldots, \gamma_{n}$ be real numbers such that for some fixed $\alpha$ and $\beta$ with $0<$ $\alpha<\beta<2$ we have $\alpha \leq \gamma_{i} \leq \beta, i=1, \ldots, n$. The bump shaping function $\psi: I^{2} \rightarrow I^{2}$ (related to $P, l, \gamma_{1}, \ldots, \gamma_{n}$ ) is defined as follows.

Let $x \in I$. If $b(x)=0$, we define $\psi(x, y)=(x, y)$. If $b(x) \neq 0$, there is a unique $i$ with $x_{i-1} \leq x \leq x_{i}$. We define

$$
\psi(x, y)= \begin{cases}\left(x, \gamma_{i} y\right), & \text { for } 0 \leq y \leq b(x), \\ (x, 2 b(x))-(2 b(x)-y)\left(2-\gamma_{i}\right), & \text { for } b(x) \leq y \leq 2 b(x) \\ (x, y), & \text { for } 2 b(x) \leq y \leq 1\end{cases}
$$

The mapping $\psi$ is a homeomorphism and $d\left(\psi\left(x, y_{1}\right), \psi\left(x, y_{2}\right)\right) \geq$ $\left|y_{1}-y_{2}\right| \min \{\alpha, 2-\beta\}$, for all $x, y_{1}, y_{2}$ and $d(\psi, i d) \leq \max \{|\beta-1|,|\alpha-1|\}$ in the supremum metric.

Proof. The lemma is proved by a straightforward computation. Note that $\{(x, 2 b(x)) \mid x \in I\}$-the graph of $2 b$-is pointwise fixed under $\psi$ and that $\psi$ is the identity above the graph of $2 b$.

3.6. Final part of the proof of Theorem 3.2. Let $\varphi$ be the map defined by Lemma 3.4. Define

$$
\phi_{1}(x, y)=(\varphi(x), y), \quad(x, y) \in I^{2} .
$$

The map $\phi_{1}$ is a homeomorphism of $I^{2}$ onto itself. The action of $\phi_{2}$ will consist of hair stretching and shrinking in the sosha $\phi_{1}\left(E_{1}\right)$. It is to be noted that $\phi_{1}$ carries the partitions $P_{n}^{1}$ to $P_{n}^{2}, n \in \mathscr{N}$. The map $\phi_{2}$ will be defined as the limit of homeomorphisms $H_{n}, n \in \mathscr{N}$, that keep the first coordinate fixed. Let $H_{0}=$ id and suppose that $H_{0}, \ldots, H_{n}$ have been defined. Let $\psi_{n}=H_{n} \circ \cdots \circ H_{0}$. Observe that $\psi_{n}\left(\phi_{1}\left(E_{1}\right)\right)$ is a sosha and let $l_{n}$ be its length function. Let $H_{n+1}$ be the bump shaping function related to $P_{n}^{2}, l_{n}$ and $\frac{M_{n+1, i}^{2}}{M_{n, j}^{2}}$ : $\frac{M_{n+1, i}^{1}}{M_{n, j}^{1}}\left(\right.$ where $j$ is determined by $i$ ). Define $\psi_{n+1}=H_{n+1} \circ \psi_{n}$. Note that

$$
d\left(\psi_{n+1}, \psi_{n}\right)=d\left(H_{n+1}, i d\right) \leq \frac{1}{(n+2)^{2}} .
$$

The inequality in the previous formula follows from (4) in 3.3. It follows that $\left(\psi_{n}\right)$ is a Cauchy sequence in the space of continuous function of $I^{2}$ into itself with the maximum metric. Its limit is denoted by $\phi_{2}$. That $\phi_{2}$ is injective, whence a homeomorphism, follows from the fact that

$$
d\left(H_{n+1}\left(x, y_{1}\right), H_{n+1}\left(x, y_{2}\right)\right) \geq\left|y_{1}-y_{2}\right|\left(1-\frac{1}{(n+2)^{2}}\right), \quad n \in \mathscr{N} \text {. }
$$

We omit the easy proofs of the following corollaries. 
3.7. Corollary. In a hairy arc $X$ there are five homeomorphism types of points. Any two points of the same type can be mapped into each other by a homeomorphism of $X$. The list of types for a sosha is as follows:

$$
\begin{aligned}
& P_{1}=\{(0,0),(1,0)\}, \\
& P_{2}=\{(x, 0) \mid x \in(0,1) \text { and } l(x)=0\}, \\
& P_{3}=\{(x, 0) \mid x \in(0,1) \text { and } l(x)>0\}, \\
& P_{4}=\{(x, l(x)) \mid x \in(0,1) \text { and } l(x)>0\}, \\
& P_{5}=\{(x, y) \mid x \in(0,1), 0<y<l(x)\} .
\end{aligned}
$$

Any point of $P_{1} \cup P_{2} \cup P_{3}$ is called a base point, any point of $P_{4}$ is called an endpoint. The points of $P_{2}$ and $P_{1}$ are called hairless base-points.

3.8. Corollary. Every base point in the hairy arc $X$ has arbitrarily small neighborhoods which are homeomorphic to $X$.

3.9. A continuum $X$ is called decomposable provided it can be written as the union of two proper subcontinua and hereditarily decomposable provided all of its subcontinua are decomposable. It is known that indecomposable continua exist (for example a solenoid). A dendroid is a hereditarily decomposable uniquely arcwise connected continuum. A fan is a dendroid containing exactly one branch point (called the vertex of the fan). Let $a, b \in X$, where $X$ is a dendroid. We denote by $[a, b]$ the unique arc in $X$ joining $a$ to $b$. A dendroid $X$ is (topologically) smooth (with respect to $p \in X$ ) provided there exists a point $p \in X$ such that for each sequence $x_{i} \rightarrow x_{0} \in X, \operatorname{Lim}\left[x_{i}, p\right]=\left[x_{0}, p\right]$. An endpoint $e$ of a dendroid $X$ is a point which is an endpoint of every arc in $X$ containing $e$. A comb is a smooth dendroid $X$ containing an arc $B$ (called the base of the comb) such that (1) the closure of every component $C$, called a hair of the comb, of $X \backslash B$ is an arc, (2) the intersection of the closures of any two hairs is empty, and (3) the set $X \backslash B$ is dense in $X$.

In $\S 2$ we defined a hairy arc as any topological image of a sosha. It follows from $\S 3$ and $\S 4$ that all hairy arcs are homeomorphic and that all oshas are equivalently embedded in the plane. In this section we will provide an internal characterization of hairy arcs. These results can also be obtained for brushes and hairy circles. We will first recall a result from [BO].

3.10. Theorem. Let $X$ and $Y$ be smooth fans with a dense set of endpoints. Then $X$ is homeomorphic to $Y$.

We have the following result:

3.11. Theorem. Let $X$ be a topological space. Then the following are equivalent:

(1) $X$ is a hairy arc,

(2) $X$ is a comb with base $B$ and order $<$ on $B$ such that for each nondegenerate hair $h_{x}$ attached at $x$, there exist hairs $\left\{h_{l_{i}}\right\}$ and $\left\{h_{r_{i}}\right\}$ such that $\lim h_{l_{i}}=h_{x}=\lim h_{r_{i}}$ and $l_{i}<x<r_{i}$ for each $i$.

Proof. (1) $\rightarrow(2)$. Since every ha is homeomorphic with a sosha, (2) is obviously satisfied.

$(2) \rightarrow(1)$. We must produce a homeomorphism $h$ between $X$, satisfying (2), and a sosha. Let $\mu: 2^{X} \rightarrow[0,1]$ be a Whitney function (i.e., a continuous 
function from the space of closed subsets with the Hausdorff metric to $[0,1]$ such that $\mu(\{x\})=0$ for every singleton $\{x\}$ and $A \varsubsetneqq B \Rightarrow \mu(A)<\mu(B)$, see [N]). Let $B$ be the base of the comb $X$ and let $g: B \rightarrow\{(x, 0) \mid 0 \leq x \leq$ 1) $\subset \mathscr{R}^{2}$ be a homeomorphism. Extend $g$ over all of $X$ as follows: for each $x \in X$, there exists a unique (possibly degenerate) irreducible arc $A_{x}$ joining $x$ to $B$. Let $A_{x} \cap B=a_{x}$. Put

$$
h(x)=\left(g\left(a_{x}\right), \mu\left(\left[a_{x}, x\right]\right)\right) .
$$

Then it follows easily from (2) and the properties of a Whitney function that $h$ is an embedding of $X$ into $I^{2}$. Since $h(X) \subset I^{2}$ is clearly a sosha, the proof is complete.

3.12. Corollary. Let $X$ and $Y$ be any two spaces satisfying (2). Then $X$ is homeomorphic to $Y$.

The following corollary follows in part from the results in $\S 4$.

3.13. Corollary. Let $\varphi_{i}: X \hookrightarrow \mathscr{R}^{2}$ be two embeddings of any space satisfying (2) such that all hairs are embedded on the same side of the arc $\varphi_{i}(B)(i=1,2)$, where $B$ is the base of $X$. Then there exists a homeomorphism $\psi: \mathscr{R}^{2} \rightarrow \mathscr{R}^{2}$ such that $\psi\left(\varphi_{1}(X)\right)=\varphi_{2}(X)$ and $\psi \mid \varphi_{1}(X)=\varphi_{2} \circ \varphi_{1}^{-1}$.

\section{TAMENESS OF THE ONE-SIDED HAIRY ARC}

In this section we study oshas, planar one-sided hairy arcs. For convenience, we shall work in the square $I^{2}$ rather than in the plane. An osha $X$ is the image of a sosha under a topological embedding in $\mathscr{R}^{2}$ such that all hairs are attached to the same side of the base. After applying a homeomorphism of $\mathscr{R}^{2}$, we can always assume that $X \subset I^{2}$, the base $B$ of $X$ coincides with $\{(x, 0) \mid 0 \leq x \leq 1\}$ and $X \cap \partial I^{2}=B$. We will refer to such an embedding as a regular embedding of an osha into the plane. Since all soshas are homeomorphic by Theorem 3.2, all oshas are homeomorphic as well. The main result is that all oshas are ambiently homeomorphic, as is stated in the following theorem.

4.1. Theorem. Suppose $X$ and $Y$ are oshas. Let $\varphi: X \rightarrow Y$ be a homeomorphism. Then there exists a homeomorphism $\Phi: S^{2} \rightarrow S^{2}$ which is an extension of $\varphi$, i.e., $\Phi \mid X=\varphi$.

For the proof of the theorem we may assume that $X$ is a sosha, as can be shown in the following way. Suppose that the oshas $X$ and $Y$ are defined by topological embeddings $\psi_{E}$ and $\psi_{F}$ of the soshas $E$ and $F$ respectively. In the diagram below, the map $\varphi_{\circ}$ is defined by commutativity of the diagram: $\varphi_{\circ}=\psi_{F}^{-1} \circ \varphi \circ \psi_{E}$

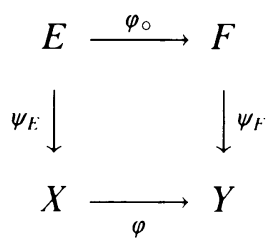

Instead of defining the extension $\phi$ of $\varphi$ directly we can as well define extensions of $\psi_{E}, \psi_{F}$ and $\varphi_{\circ}$. For the latter maps the domain is a sosha. 
The authors would like to thank Cor Baayen for pointing out this simplification of the extension problem.

4.2. Notation. Throughout this section we shall assume that each osha is regularly embedded in $I^{2}$ and use $h_{x}$ to denote the (possibly degenerate) hair attached at the point $x$ of the base. In a sosha it is the set $\{(x, y) \mid 0 \leq y \leq l(x)\}$.

The proof of Theorem 4.1, to be presented in 4.6, requires some preparation. Several ideas from the theory of prime ends are used.

4.3. Prime ends. Here we collect a few facts from the theory of prime ends. For details the reader is referred to [Ma] and the references therein. Let $X$ be a regularly embedded osha in $I^{2}$. The complementary component of $X$ in $\operatorname{Int}\left(I^{2}\right)$, denoted by $U$, is a connected and simply connected open set, because $X$ is homotopically trivial. Note that $\partial U=X \cup \partial I^{2}$. A crosscut (of $U$ ) is an arc $C$ with endpoints $a$ and $b$ such that $C \cap \partial U=\{a, b\}$. The crosscut $C$ is usually denoted by $[a, b]$. A crosscut divides $U$ into two disjoint domains (connected open sets), which are referred to as the complementary domains of $C$. For convenience a fixed point of $U$, say $\omega$, is selected and only those crosscuts are considered that do not pass through $\omega$. The complementary domain which contains $\omega$ is called the unbounded complementary domain, while the other domain is said to be bounded. A chain of crosscuts (of $U$ ) is a sequence $\left(C_{n}\right)$ of crosscuts such that:

(i) $C_{n}$ effects a separation between $C_{n+1} \cap U$ and $\omega$ in $U, n \in \mathcal{N}$;

(ii) $C_{n} \cap C_{m}=\varnothing$ for all $n \neq m$ in $\mathscr{N}$;

(iii) $C_{n} \rightarrow p \quad\left(C_{n}\right.$ converges to $\left.p\right)$ for some $p \in X$.

Two chains of crosscuts $\left(C_{n}\right)$ and $\left(D_{n}\right)$ are equivalent provided each crosscut $C_{m}$ effects a separation in $U$ between the point $\omega$ and all but finitely many $D_{n}$ and, similarly, each $D_{m}$ effects a separation in $U$ between $\omega$ and all but finitely many $C_{n}, \quad m \in \mathscr{N}$. By this equivalence relation, the collection of all chains of crosscuts is partitioned in prime ends. The principal set of a prime end $E$ is the set

$$
P(E)=\left\{p \in \partial U \mid C_{n} \rightarrow p \text { for some chain }\left(C_{n}\right) \in E\right\} .
$$

4.4. Lemma. Let $X \subset I^{2}$ be a regularly embedded osha and $U=\operatorname{Int}\left(I^{2}\right) \backslash X$. Then, for any prime end $E$, the principal set $P(E)$ consists of a single point, which is denoted by $p_{E}$. Moreover, $p_{E}$ is either an endpoint of a hair, a point of $\partial I^{2} \backslash X$ or a hairless base point. Conversely, every endpoint of a hair, every point of $\partial I^{2} \backslash X$ and every hairless base point is the principal set of some prime end and hence arcwise accessible from $U$.

Proof. The lemma follows easily for prime ends associated with points of $\partial I^{2} \backslash X$. Hence suppose that $\left(C_{n}\right)$ and $\left(D_{n}\right)$ are equivalent chains of the prime end $E$ such that $C_{n} \rightarrow p$ and $D_{n} \rightarrow q$, where $p, q \in X$. The endpoints $a_{n}$ and $b_{n}$ of the crosscut $C_{n}$ are both accessible from $U, n \in \mathscr{N}$. From the property (3) in Definition 2.1 it follows that $a_{n}$ as well as $b_{n}$ are an endpoint of a hair or a hairless base point. Suppose $p \in h_{x}, a_{n} \in h_{\alpha_{n}}$ and $b_{n} \in h_{\beta_{n}}, n \in \mathcal{N}$, the hairs possibly being degenerate. As $C_{n}$ is a separation between $p$ and $\omega$, the points $\alpha_{n}$ and $\beta_{n}$ are at different sides of $x$ for each $n$ ("sides" refers to the natural order on the base). Moreover, $\alpha_{n} \rightarrow x$ and $\beta_{n} \rightarrow x$. Since $X$ is smooth, $p$ must be the endpoint of $h_{x}$. Similarly, $q$ is the endpoint of a (possibly degenerate) hair $h_{y}$. An analysis of $\left(D_{n}\right)$ similar to that of $\left(C_{n}\right)$ leads 
to the conclusion that $x=y$, whence $p=q$. Now we show that each endpoint of a hair is the limit of a chain of crosscuts. The same result for hairless base points can be proved in a similar fashion. That these points are arcwise accessible from $U$ then follows from prime end theory.

Let $e$ be an endpoint of the hair $h_{x}$. Without loss of generality, we may assume that $h_{x}$ (and the base $B$ ) of $X$ is a straight line in the square. Let $<$ denote a natural order on $B$. Choose $\varepsilon_{1}$, such that $S\left(e, 2 \varepsilon_{1}\right) \cap B=\varnothing$. Here $S\left(e, 2 \varepsilon_{1}\right)$ denotes the circle with center $e$ and radius $2 \varepsilon_{1}$. Let $\prec$ denote an order on the arc $S_{1}=S\left(e, \varepsilon_{1}\right) \backslash h_{x}$ such that for any $a \in h_{c} \cap S_{1}, b \in$ $h_{d} \cap S_{1}$, where $c<x<d$ and $|c-d|$ sufficiently small, we have $a \prec b$. Let $a_{1}=\max \left\{z \in S_{1} \mid z \in h_{p}\right.$ and $\left.p<x\right\}$ and let $b_{1}=\min \left\{z \in S_{1} \mid z \succ a_{1}, z \in\right.$ $h_{q}$ and $\left.x<q\right\}$. Then $Q_{1}=\left[a_{1}, b_{1}\right] \subset S_{1}$ is a crosscut such that $h_{x}$ is contained in the bounded complementary domain of $Q_{1}$. Assume that $a_{1} \in h_{\alpha_{1}}$ and $b_{1} \in h_{\beta_{1}}$. Then $\alpha_{1}<x<\beta_{1}$. Choose $\varepsilon_{2}<\varepsilon_{1} / 4$ such that, for any $y \in B$ with $h_{y} \cap S\left(e, 2 \varepsilon_{2}\right) \neq \varnothing, \alpha_{1}<y<\beta_{1}$. By repeating the above argument, we find a crosscut $Q_{2} \subset S_{2}$ such that $h_{x}$ is contained in the bounded complementary domain of $Q_{2}$ and $Q_{2}$ is contained in the bounded complementary domain of $Q_{1}$. In this way we can construct a chain of crosscuts $\left(Q_{n}\right)$ with $Q_{n} \rightarrow e$. The proof is complete.

Having established the arcwise accessibility of the endpoints of hairs and of the hairless base points of the oshas, we can now prove the key lemma about the existence of sufficiently many small crosscuts.

Lemma 4.5. Let $\varphi: X \rightarrow Y$ be regular topological embedding of a sosha $X$ onto an osha $Y$ in $I^{2}$. Then

(1) for every sequence of crosscuts $C_{n}=\left[a_{n}, b_{n}\right]$ of $I^{2} \backslash X$ with $\operatorname{diam}\left(C_{n}\right)$ $\rightarrow 0$, there exists a sequence of crosscuts $D_{n}=\left[\varphi\left(a_{n}\right), \varphi\left(b_{n}\right)\right]$ of $I^{2} \backslash Y$ with $\operatorname{diam}\left(D_{n}\right) \rightarrow 0$.

(2) for every sequence of crosscuts $C_{n}=\left[a_{n}, b_{n}\right]$ of $I^{2} \backslash Y$ with $\operatorname{diam}\left(C_{n}\right) \rightarrow$ 0 , there exists a sequence of crosscuts $D_{n}=\left[\varphi^{-1}\left(a_{n}\right), \varphi^{-1}\left(b_{n}\right)\right]$ of $I^{2} \backslash X$ with $\operatorname{diam}\left(D_{n}\right) \rightarrow 0$.

Proof. We shall only proof (1). The proof of (2) is similar, but somewhat easier. The proof is by way of contradiction. Assume that (1) fails. Then there exist $\varepsilon$, $0<\varepsilon<1 / 2$, and a sequence $\left(C_{n}\right), C_{n}=\left[a_{n}, b_{n}\right]$, of crosscuts of $I^{2} \backslash X$ such that $\operatorname{diam}\left(C_{n}\right) \rightarrow 0$ but, for every $n$ and every crosscut $D_{n}=\left[\varphi\left(a_{n}\right), \varphi\left(b_{n}\right)\right]$, $\operatorname{diam}\left(D_{n}\right) \geq \varepsilon$. It is to be noted that crosscuts $\left[\varphi\left(a_{n}\right), \varphi\left(b_{n}\right)\right]$ exist by Lemma 4.4. Without loss of generality we may assume $C_{n} \rightarrow c$, for a point $c$ of some possibly degenerate hair $h_{\gamma}$ of $X$, where $\gamma \in B_{X}$, the base of $X$. Suppose that $a_{n}\left(b_{n}\right)$ is an endpoint of the hair $h_{\alpha_{n}}\left(h_{\beta_{n}}\right.$, respectively). We may assume that the base $B_{Y}$ of the osha $Y$ and the hair $\varphi\left(h_{\gamma}\right)$ are straight line segments in $I^{2} \backslash\{\omega\}$. Consider an open neighborhood $W$ of $d=\varphi(c)$ such that $\omega \in I^{2} \backslash \mathrm{cl}(W)$ and the boundary of $W$ is a topological circle $S$ with center $d$ and radius $\mu<\varepsilon / 2$. As $\varphi$ is uniformly continuous there exists a $\delta$ such that $d(\varphi(y), \varphi(z))<\mu / 8$ for any $y, z$ with $d(y, z)<\delta$. As $X$ is a sosha, there is a rectangular neighborhood $V$ of $c$ with diameter $\delta$ which has the following additional properties: (1) every hair intersected with $V$ is an interval, (2) each hair $h_{z}$ with $z \in \bigcup_{n}\left[\alpha_{n}, \beta_{n}\right]$ that enters $V$ does not leave $V$ (i.e., if $(z, s) \in V, \alpha_{n}<z<\beta_{n}$, then $(z, u) \in V$ for all $\left.s<u \leq l(z)\right)$ 
and each hair intersects $\partial V$ in at most two points. Let $W_{0}$ be a neighborhood of $d$ with radius less than $\mu / 8$ such that $\varphi^{-1}\left(W_{0}\right) \subset V$. As $C_{n} \rightarrow c$, the straight line segment $J_{n}$ between $\varphi\left(a_{n}\right)$ and $\varphi\left(b_{n}\right)$ as well as its endpoints lie in $W_{0}$ for $n$ sufficiently large. By Lemma 4.4, the points $\varphi\left(a_{n}\right)$ and $\varphi\left(b_{n}\right)$ can be joined with $\omega$ by arcs denoted by $\left[\omega, \varphi\left(a_{n}\right)\right]$ and $\left[\omega, \varphi\left(b_{n}\right)\right]$, respectively. By assumption it is not possible to make a crosscut $D_{n}=\left[\varphi\left(a_{n}\right), \varphi\left(b_{n}\right)\right]$ by piecing together parts of the circle $S$ and portions of the arcs $\left[\omega, \varphi\left(a_{n}\right)\right]$ and $\left[\omega, \varphi\left(b_{n}\right)\right]$ that lie in $W$. It follows that for some $t_{n}$ and some $y_{n} \in \varphi\left(h_{t_{n}}\right)$, we have $y_{n} \in W_{0}$, but the hair $\varphi\left(h_{t_{n}}\right)$ leaves $W$ after $y_{n}$ (and hits $S$ ). Then $\varphi^{-1}\left(y_{n}\right) \in V$. By the choice of $V, t_{n} \in B_{X} \backslash\left(\bigcup_{m}\left[\alpha_{m}, \beta_{m}\right]\right)$. By again using the fact that we cannot piece together the required cross cuts from $S$ and the arcs $\left[\omega, \varphi\left(a_{n}\right)\right]$ and $\left[\omega, \varphi\left(b_{n}\right)\right]$, we conclude that the hair $\varphi\left(h_{t_{n}}\right)$ must enter a bounded complementary domain of $J_{n} \cup \varphi\left(h_{\alpha_{n}}\right) \cup \varphi\left(h_{\beta_{n}}\right) \cup\left[\varphi\left(\alpha_{n}\right), \varphi\left(\beta_{n}\right)\right]$ in the complement of $W$. Let $z_{n}$ be a point of $\varphi\left(h_{t_{n}}\right)$ after $y_{n}$ in this bounded complementary domain which is not in $W$. We may assume that the sequence $\left(z_{n}\right)$ converges to a point $q$ in $[\varphi(\gamma), \varphi(c)]$. Hence, $\varphi^{-1}(q) \in[\gamma, c]$. Since $\varphi^{-1}\left(z_{n}\right) \notin V$ and $\varphi^{-1}\left(z_{n}\right)$ is a point of $h_{t_{n}}$ after $\varphi^{-1}\left(y_{n}\right), \lim \varphi^{-1}\left(z_{n}\right)=$ $\varphi^{-1}(q) \notin[\gamma, c]$. This contradiction completes the proof of the lemma.

4.6. Proof of Theorem 4.1. As has been explained earlier we may assume that $X$ is a sosha and $Y$ is an osha, $X$ has the standard form and $Y$ is regularly embedded in $I^{2}$. Put $G_{0}=\partial I^{2}, H_{0}=\partial I^{2}$, and let $\Phi_{0}: G_{0} \cup X \rightarrow H_{0} \cup Y$ be any homeomorphic extension of $\varphi$. Using Lemmas 4.4 and 4.5 one can inductively construct graphs $G_{n}$ and $H_{n}$ and homeomorphisms

$$
\Phi_{n}: X \cup G_{n} \rightarrow Y \cup H_{n}
$$

such that, for all $n$,

(1) $G_{n} \subset G_{n+1}$ and $\Phi_{n+1} \mid X \cup G_{n}=\Phi_{n}$

(2) the diameter of any complementary domain of $G_{n}$ or $H_{n}$ is less than $1 / 2^{n}$.

The construction of the graphs $G_{n}$ and $H_{n}$ follows the pattern of the classical proofs of the existence of extensions of homeomorphisms of arcs or Cantor sets. It is to be noted that for any arc $J$, which joins two points of $\partial I^{2}$ in such a way that $J \cap \partial I^{2}$ only consists of two points, the set $J \backslash\left(X \cup G_{0}\right)$ is the union of a null sequence of crosscuts of $I^{2} \backslash\left(X \cup G_{0}\right)$. By Lemma 4.5 there exists a similar null sequence of crosscuts for $I^{2} \backslash\left(Y \cup H_{0}\right)$. This null sequence of crosscuts can be used to extend the homeomorphism $\Phi_{0}$ over $X_{0} \cup G_{0} \cup J$.

Since the complementary domains of the homeomorphic graphs $G_{n}$ and $H_{n}$ are in one-to-one correspondence, in view of (2) we can define an extension $\Phi$ of

$$
\Phi_{\infty}: X \cup\left(\bigcup\left\{G_{n} \mid n \in \mathscr{N}\right\}\right) \rightarrow Y \cup\left(\bigcup\left\{H_{n} \mid n \in \mathscr{N}\right\}\right)
$$

where

$$
\Phi_{\infty}(x)=\Phi_{n}(x) \text { for } x \in X \cup\left(\bigcup G_{n}\right)
$$

The arguments leading up to the proof of Theorem 4.1 can also be used to prove a similar results for hairy circles. Hairy circles have been investigated in [FO1 and FO2]. 
4.7. Definition. A hairy circle is a hairy arc with the two endpoints of the base identified. A one-sided hairy circle is an embedding of a hairy circle into the plane such that all hairs are attached at the same side of the base circle.

4.8. Theorem. Any two hairy circles are homeomorphic. Let $\varphi: X \rightarrow Y$ be a homeomorphism between one-sided hairy circles. Then there is a homeomorphism $\Phi: S^{2} \rightarrow S^{2}$ which is an extension of $\varphi$.

\section{JULIA SETS OF HYPERBOLIC FUNCTIONS}

In this section we shall show that all Julia sets in the one-parameter families $C_{\lambda}(z)=\lambda \cosh z, 0<\lambda<\mu(\approx 0.67)$, and $S_{\lambda}(z)=\lambda \sinh z, 0<\lambda \leq$ $\mu(\approx 0.85)$, are brushes of hairy arcs. The results can be carried over to the one-parameter family $z \rightarrow \lambda i \cos z, 0<\lambda<\mu$, via the conjugating homeomorphism $z \rightarrow-i z$, and to the one-parameter family $z \rightarrow \lambda \sin z, 0<\lambda<\mu$, via the conjugating homeomorphism $z \rightarrow i z$. The techniques employed in this section are similar to those of $\S 1$, so we shall skip the details.

5.1. The family $C_{\lambda}(z), 0<\lambda<\mu$. The number $\mu$ is defined as the supremum of the parameter values $\lambda$ for which $C_{\lambda}$ has an attracting fixed point. That is $\mu$ is the solution of the system $C_{\mu}(x)=x$ and $S_{\mu}(x)=1$. The approximate solution is $\mu \approx 0.67$ and $x_{\mu} \approx 1.19$. Let $\lambda$ be a fixed real number with $0<\lambda<\mu$. The function $C_{\lambda}$ has two real fixed points, an attracting one, $q_{\lambda}$, and a repelling one, $p_{\lambda}$. With $z=x+i y$, where $x, y \in \mathscr{R}$, we have

$$
C_{\lambda}(z)=C_{\lambda}(x) \cos y+i S_{\lambda}(x) \sin y
$$

The vertical line $\{x+i t \mid t \in \mathscr{R}\}, x \neq 0$, is mapped by $C_{\lambda}$ onto an ellipse. By standard arguments it can be shown that the strip $|\operatorname{Re} z|<p_{\lambda}$ is a subset of the Fatou set of $C_{\lambda}$. Also, the lines $\left\{t+\left(k+\frac{1}{2}\right) \pi i \mid t \in \mathscr{R}\right\}, k \in \mathscr{Z}$, belong to the Fatou set. The rays $\left\{t+k \pi i \mid t \in \mathscr{R}\right.$ and $\left.t \geq p_{\lambda}\right\}, k \in \mathscr{Z}$, as well as the rays $\left\{t+k \pi i \mid t \in \mathscr{R}\right.$ and $\left.t \leq-p_{\lambda}\right\}, k \in \mathscr{Z}$ are in the Julia set of $C_{\lambda}$. By taking preimages we get interleaved collections of rays in the Julia and the Fatou set of $C_{\lambda}$.

Here is the main result concerning $C_{\lambda}$.

5.2. Theorem. Suppose $0<\lambda<\mu$. There exists a brush and a homeomorphism $\varphi$ of $B$ onto the Julia set $J\left(C_{\lambda}\right)$ of $C_{\lambda}$.

It will be convenient to define the brush $B$ as the union of two straight brushes $B^{+}$and $B^{-}$, where $B^{+}$is a subset of $(0, \infty) \times \mathscr{P}$ and $B^{-}$is a subset of $(-\infty, 0) \times \mathscr{P}$. We have the following substitute for Definition 1.5.

5.3. Definition. For each $x \in \mathscr{R}$ and $n \in \mathscr{Z}$, the square $T(x, n)$ is defined by

(i) if $x \geq 0, T(x, n)=\left\{\xi+i \eta \mid x \leq \xi \leq x+\pi,\left(n-\frac{1}{2}\right) \pi \leq \eta \leq\left(n+\frac{1}{2}\right) \pi\right\}$,

(ij) if $x<0, T(x, n)=\left\{\xi+i \eta \mid x-\pi \leq \xi \leq x,\left(n-\frac{1}{2}\right) \pi \leq \eta \leq\left(n+\frac{1}{2}\right) \pi\right\}$.

Note that $T(x, n) \cap J\left(C_{\lambda}\right) \neq \varnothing$ for all $n \in \mathscr{Z}$ and all $x$ with $|x| \geq p_{i}$. For each $x$ with $|x| \geq p_{\lambda}$ and all $n \in \mathscr{Z}$, the set $C_{\lambda}(T(x, n))$ is a "semi-annulus" the boundary of which consists of two semi-ellipses and two intervals on the imaginary axis. The radial width of the semi-annulus exceeds 22 . 
5.4. Construction of $B^{+}$and $B^{-}$; definition of $\varphi$. Let $|x| \geq p_{\lambda}$ and $\alpha \in \mathscr{P}$. Using the product representation of $\mathscr{P}$ we write $\alpha=\left(n_{0}, n_{1}, \ldots\right)$ with $n_{i} \in$ $\mathscr{Z}$. Inductively on $k$ we define real numbers $x_{k}$ and sets $R\left(x_{k}, n_{k}\right)$. We let $x_{0}=x$ and $R\left(x_{0}, n_{0}\right)=T\left(x, n_{0}\right)$. Suppose that $x_{l}$ and $R\left(x_{l}, n_{l}\right)$ have been defined for $l \leq k$. We consider five cases.

1. $R\left(x_{k}, n_{k}\right) \neq \varnothing, x_{k}>0, n_{k}$ even and there exists $\xi$ with

$$
T\left(\xi, n_{k+1}\right) \subset C_{\lambda}\left(R\left(x_{k}, n_{k}\right) .\right.
$$

2. $R\left(x_{k}, n_{k}\right) \neq \varnothing, x_{k}<0, n_{k}$ even and there exists $\xi$ with $(*)$. Note that in both case 1 and case 2

$$
C_{\lambda}\left(R\left(x_{k}, n_{k}\right)\right) \subset\{z \mid \operatorname{Re} z>0\} .
$$

In both cases we let $\xi_{m}=\min \{\xi \mid \xi$ satisfies $(*)\}$. If $\xi_{m} \geq p_{\lambda}$, we define $x_{k+1}=$ $\xi_{m}$ and $R\left(x_{k+1}, n_{k+1}\right)=T\left(x_{k+1}, n_{k+1}\right)$. If $\xi_{m}<p_{\lambda}$ we let $x_{k+1}=x_{k}$ and $R\left(x_{k+1}, n_{k+1}\right)=\varnothing$.

3. $R\left(x_{k}, n_{k}\right) \neq \varnothing, x_{k}>0, n_{k}$ odd and there exists $\xi$ with $(*)$.

4. $R\left(x_{k}, n_{k}\right) \neq \varnothing, x_{k}<0, n_{k}$ odd and there exists $\xi$ with $(*)$. In cases 3 and 4 ,

$$
C_{\lambda}\left(R\left(x_{k}, n_{k}\right)\right) \subset\{z \mid \operatorname{Re} z<0\} .
$$

We put $\xi_{m}=\max \{\xi \mid \xi$ satisfies $(*)\}$. If $\xi_{m} \leq-p_{\lambda}$, we define $x_{k+1}=\xi_{m}$ and $R\left(x_{k+1}, n_{k+1}\right)=T\left(x_{k+1}, n_{k+1}\right)$. Otherwise, $x_{k+1}=x_{k}$ and $R\left(x_{k+1}, n_{k+1}\right)=$ $\varnothing$.

5. If none of the cases 1-4 applies, we let $x_{k+1}=x_{k}$ and $R\left(x_{k+1}, n_{k+1}\right)$ $=\varnothing$. The straight brushes $B^{+}$and $B^{-}$are defined by $B^{+}=\{(x, \alpha) \mid x \geq$ $p_{\lambda}$ and $R\left(x_{k}, n_{k}\right) \neq \varnothing$, for all $\left.k\right\}$ and $B^{-}=\left\{(x, \alpha) \mid x \leq-p_{\lambda}\right.$ and $R\left(x_{k}, n_{k}\right) \neq$ $\varnothing$, for all $k\}$. For each $(x, \alpha) \in B^{+} \cup B^{-}$, we let

$$
\varphi(x, \alpha)=\bigcap\left\{C_{\lambda}^{-k}\left(R\left(x_{k}, n_{k}\right)\right) \mid k=0,1,2, \ldots\right\} .
$$

Note that $\varphi$ is well defined, because $\left|C_{\lambda}^{\prime}(z)\right|>1$ on the set $\left\{z|| \operatorname{Re} z \mid>p_{\lambda}\right\}$. The proof that $B^{+}$and $B^{-}$are straight brushes and $\varphi$ is an injective continuous map of $B^{+} \cup B^{-}$into $J\left(C_{\lambda}\right)$ is similar to the proof of Lemma 1.7.

5.5. Construction of $\psi=\varphi^{-1}$. Suppose that $z \in J\left(C_{\lambda}\right)$. Note that $\operatorname{Re}\left(C_{\lambda}^{k}(z)\right)$ $\neq 0$. The itinerary $n(z)=\left(n_{0}, n_{1}, \ldots\right)$ is defined by the conditions,

$$
\left(n_{k}-\frac{1}{2}\right) \pi<\operatorname{Im} C_{\lambda}^{k}(z)<\left(n_{k}+\frac{1}{2}\right) \pi, \quad k \in \mathscr{Z} .
$$

Inductively on $k$, we shall define $R_{l}^{k}$ for $0 \leq 1 \leq k$. First we define $R_{k}^{k}$ for all $k \in \mathscr{N}$. If $\operatorname{Re}\left(C_{\lambda}^{k}(z)\right)>0$, we choose $u$ minimal with respect to the properties $u \geq p_{\lambda}$ and $C_{\lambda}^{k}(z) \in T\left(u, n_{k}\right)$. If $\operatorname{Re}\left(C_{\lambda}^{k}(z)\right)<0$, we choose $u$ maximal with respect to the properties $u \leq-p_{\lambda}$ and $C_{\lambda}^{k}(z) \in T\left(u, n_{k}\right)$. We define $R_{k}^{k}=T\left(u, n_{k}\right)$. For $k=1,2, \ldots$, we define $R_{i}^{k}$, for $i=k-1$ to $i=0$, inductively. Suppose that $R_{l}^{k}$ has been defined. Observe that either

$$
R_{l-1}^{k-1} \subset\{w \mid \operatorname{Re} w>0\}
$$

or

$$
R_{l-1}^{k-1} \subset\{w \mid \operatorname{Re} w<0\}
$$


We define $R_{l-1}^{k}=T\left(v, n_{k-1}\right)$, where in case $1 v$ is maximal and in case 2 $v$ is minimal with respect to $R_{l}^{k} \subset C_{\lambda}\left(R_{l-1}^{k}\right)$. Note that this definition ensures that the square $R_{l}^{k}$ hits the "inner" semi-ellipse of the semi-annulus $C_{\lambda}\left(R_{l-1}^{k}\right)$ in exactly one point. For each $k \in \mathscr{N}$, we define $x(k)$ by the condition $R_{0}^{k}=T\left(x(k), n_{0}\right)$. It is to be noted that $z \in R_{0}^{k}$, for each $k \in \mathcal{N}$. If Re $z>0$, we have

$\operatorname{Re} z-\pi \leq x_{0} \leq \operatorname{Re} z$, and $x(0) \leq x(k) \leq x(k+1) \leq \operatorname{Re} z+\pi$, for all $k$.

If $\operatorname{Re} z<0$, we have

$\operatorname{Re} z \leq x(0) \leq \operatorname{Re} z+\pi$, and $\operatorname{Re} z-\pi \leq x(k+1) \leq x(k) \leq x_{0}$, for all $k$.

Finally we define

$$
x^{\infty}=\lim _{k \rightarrow \infty} x(k) \text { and } \psi(z)=\left(x^{\infty}, n(z)\right) .
$$

The proof of Theorem 5.2 can be completed in the same way as in Lemmas 1.9 and 1.10 .

The discussion of 1.12 can be generalized in a straightforward manner. Compactifying $C$ by $\mathscr{R}^{*} \times \mathscr{R}^{*}$, we get a compactification of $B=B^{+} \cup B^{-}$by taking the closure of $B$ in $\mathscr{R}^{*} \times \mathscr{R}^{*}$. In this way $B$ is compactified by the union of two hairy arcs. In a similar way as in 1.12 the Julia set $J\left(C_{\lambda}\right)$ can be compactified by the union of two hairy arcs. Identification of the set $\mathscr{R}^{*} \times\{\infty\}$ to one point yields compactifications of $B^{+} \cup B^{-}$and $J\left(C_{\lambda}\right)$ by just one hairy arc.

We now discuss the family $S_{\lambda}(z), 0<\lambda \leq \nu$. As the discussion is parallel to that of the family $C_{\lambda}(z)$, the exposition will be even more sketchy.

5.6. The family $S_{\lambda}(z), 0<\lambda \leq \nu$. The number $\nu$ is defined by the condition $\nu \sinh (1)=1 ; \nu \approx 0.85$. Let $\lambda$ be a fixed real number with $0<\lambda \leq \nu$. The function $S_{\lambda}$ has three real fixed points. The origin is an attracting fixed point. The imaginary axis is in its basin of attraction and so are the lines $\left\{t+\left(k+\frac{1}{2}\right) \pi i \mid t \in \mathscr{R}\right\}, k \in Z$. On the positive real axis there is a unique fixed point $p_{\lambda}$. Elementary considerations show that $p_{\lambda}$ is a repelling fixed point. Similarly, $-p_{\lambda}$ is a repelling fixed point of $S_{\lambda}$. It can be seen that the rays $\left\{t+k \pi i \mid t \in \mathscr{R}, t \geq p_{\lambda}\right\}$ and $\left\{t+k \pi i \mid t \in \mathscr{R}, t \leq-p_{\lambda}\right\}, k \in \mathscr{Z}$, are in the Julia set $J\left(S_{\lambda}\right)$. Writing $z=x+i y$, we get

$$
S_{\lambda}(z)=S_{\lambda}(x) \cos y+i C_{\lambda}(x) \sin y .
$$

The vertical line $\{x+i t \mid t \in \mathscr{R}\}, x \neq 0$, is mapped by $S_{\lambda}$ onto an ellipse. By Schwarz's lemma it follows that $\left\{z|| \operatorname{Re} z \mid<p_{\lambda}\right\}$ is in the basin of attraction of 0 .

Remark. In contrast with the situation in 5.1, there is no "dynamical" argument for imposing the upper bound $\nu$ instead of using the natural upper bound 1 . However by imposing this constraint on $\lambda$, we have an easy way to guarantee that $\left|S_{\lambda}^{\prime}(z)\right| \geq 1$ for $|z| \geq p_{\lambda}$. There is the following result.

5.7. Theorem. Suppose $0<\lambda<\nu$. There exists a brush $B$ and a homeomorphism $\varphi$ of $B$ onto the Julia set $J\left(S_{\lambda}\right)$ of $S_{\lambda}$.

As before, $B=B^{+} \cup B^{-}$and we shall consider $B^{+}$as a subset of $(0, x) \times \mathscr{P}$ and $B^{-}$as a subset of $(-\infty, 0) \times \mathscr{P}$. In the following definition, which is in 
the same spirit as Definitions 1.5 and 5.3, we use rectangles instead of squares in order to make the analogues of 5.3 and 5.5 work.

5.8. Definition. Let $c$ be a real constant such that $S_{\lambda}\left(\frac{c}{2}\right) \geq 4 c \geq 4 \pi$. For each $n \in \mathscr{Z}$ and each $x \in \mathscr{R}$, the rectangle $T_{c}(x, n$,$) is defined by$

(i) if $x \geq 0, T_{c}(x, n)=\left\{\xi+i \eta \mid x \leq \xi \leq x+c,\left(n-\frac{1}{2}\right) \pi \leq \eta \leq\left(n+\frac{1}{2}\right) \pi\right\}$;

(ij) if $x<0, T_{c}(x, n)=\left\{\xi+i \eta \mid x-c \leq \xi \leq x,\left(n-\frac{1}{2}\right) \pi \leq \eta \leq\left(n+\frac{1}{2}\right) \pi\right\}$.

Observe that $T_{c}(x, n) \cap J\left(S_{\lambda}\right) \neq \varnothing$, for all $n \in Z$ and all $x$ with $|x| \geq p_{\lambda}$. For each $x$ with $|x| \geq p_{\lambda}$ and all $n \in \mathscr{Z}$, the set $S_{\lambda}\left(T_{c}(x, n)\right)$ is a semi-annulus which is bounded by two semi-ellipses and two intervals on the imaginary axis. The differences between the length of the axes exceed $16 c$.

5.9. Construction of $B^{+}$and $B^{-}$; definition of $\varphi$. Let $|x| \geq p_{\lambda}$ and $\alpha=$ $\left(n_{0}, n_{1}, \ldots\right) \in \mathscr{P}$. Inductively on $k$ we define real numbers $x_{k}$ and sets $R\left(x_{k}, n_{k}\right)$. Let $x_{0}=x$ and $R\left(x_{0}, n_{0}\right)=T_{c}\left(x, n_{0}\right)$. Suppose that $x_{l}$ and $R\left(x_{l}, n_{l}\right)$ have been defined for $l \leq k$. There are five cases to consider.

1. $R\left(x_{k}, n_{k}\right) \neq \varnothing, x_{k}>0, n_{k}$ even and there exists $\xi$ with

$$
T_{c}\left(\xi, n_{k+1}\right) \subset S_{\lambda}\left(R\left(x_{k}, n_{k}\right)\right) \text {. }
$$

2. $R\left(x_{k}, n_{k}\right) \neq \varnothing, x_{k}<0, n_{k}$ odd and there exists $\xi$ with $(*)$.

3. $R\left(x_{k}, n_{k}\right) \neq \varnothing, x_{k}>0, n_{k}$ odd and there exists $\xi$ with $(*)$.

4. $R\left(x_{k}, n_{k}\right) \neq \varnothing, x_{k}<0, n_{k}$ even and there exists $\xi$ with $(*)$.

5. None of 1 through 4 applies. The definition of $x_{k+1}$ is as in 5.4 and in case 1 through 4 we let $R\left(x_{k+1}, n_{k+1}\right)=T_{c}\left(x_{k+1}, n_{k+1}\right)$. The proof continues as in 5.4.

5.10. Construction of $\varphi^{-1}$. The construction of $\varphi^{-1}: J\left(S_{\lambda}\right) \rightarrow B^{+} \cup B^{1}$ is almost verbatim as in 5.5. We only have to substitute $S_{\lambda}$ for $C_{\lambda}$ and $T_{c}$ for $T$.

In the exact same way as in 5.5 it follows that the Julia set $J\left(S_{\lambda}\right)$ can be compactified by a hairy arc.

\section{REFERENCES}

[BO] W. T. Bula and L. G. Oversteegen, A characterization of smooth Cantor bouquets, Proc. Amer. Math. Soc. 108 (1990), 529-534.

[C] W. J. Charatonik, The Lelek fan is topologically unique, Houston J. Math. 15 (1989), 27-34.

[D1] R. L. Devaney, Julia sets and bifurcation diagrams for exponential maps, Bull. Amer. Math. Soc. 11 (1984), 167-171.

[D2] The structural instability of $\operatorname{Exp}(z)$, Proc. Amer. Math. Soc. 94 (1985), 545-548.

[D3] _ An introduction to chaotic dynamical systems, Benjamin, Menlo Park, Calif., 1986.

[D4] _,$e^{z}$, dynamics and bifurcations, Internat. J. Bifurcations and Chaos 1 (1991), 287308.

[DG] R. L. Devaney and L. R. Goldberg, Uniformization of attracting basins for exponential maps, Duke Math. J. 55 (1987), 253-266.

[DGH] R. L. Devaney, L. R. Goldberg and J. H. Hubbard, A dynamical approximation of the exponential by polynomials, Preprint.

[DKe] R. L. Devaney and L. Keen, eds., Chaos and fractals, Proc. Sympos. Appl. Math., vol. 39. Amer. Math. Soc. Providence, R.I., 1989.

[DKr] R. L. Devaney and M. Krych, Dynamics of $\exp (z)$. Ergodic Theory Dynamical Systems 4 (1984), 35-52. 
[DT] R. L. Devaney and F. Tangerman, Dynamics of entire functions near the essential singularity, Ergodic Theory Dynamical Systems 6 (1986), 498-503.

[EL] A. È. Eremenko and M. Yu. Ljubich, Iterates of entire functions, Soviet Math. Dokl. 30 (1984), 592-594.

[FO1] R. J. Fokkink and L. G. Oversteegen, An example related to the Birkhoff Conjecture, Preprint, 1989.

[FO2] A recurrent non-rotational homeomorphism on the annulus, Trans. Amer. Math. Soc. 333 (1992), 865-875.

[G] L. R. Goldberg, Structural stability in the family $\lambda e^{z}$ (in preparation).

[K] C. Kuratowski, Topologie. I, II, PWN, Warsaw, 1958, 1961.

[L] A. Lelek, On plane dendroids and their endpoints in the classical sense, Fund. Math. 49 (1961), 301-319.

[Ma] J. C. Mayer, An explosion point for the set of endpoints of the Julia set of $\lambda \exp (z)$, Ergodic Theory Dynamical Systems 10 (1990), 177-183.

[McM] C. McMullen, Area and Hausdorff dimension of Julia sets of entire functions, Trans. Amer. Math. Soc. 300 (1987), 329-342.

[Mi] M. Misiurewicz, On iterates of $e^{z}$, Ergodic Theory Dynamical Systems 1 (1981), 103-106.

[N] S.B. Nadler, Jr., Hyperspaces of sets, Monographs in Pure and Appl. Math., Vol. 49, Marcel Dekker, New York and Basel, 1978.

[P] G. Piranian, The boundary of a simply connected domain, Bull. Amer. Math. Soc. 64 (1958), 45-55.

Faculteit Wiskunde en Informatica, TUdelft, Postbus 5031, 2600 Ga Delft, The NETHERLANDS

E-mail address: aarts@dutiaw3.tudelft.nl

Department of Mathematics, University of Alabama at Birmingham, Birmingham, AlABAMA 35294

E-mail address: overstee@math.uab.edu 